\title{
Optimal Clustering with Missing Values
}

\author{
Shahin Boluki* \\ Department of Electrical and Computer Engineering \\ Texas A\&M University \\ College Station, Texas \\ s.boluki@tamu.edu \\ Xiaoning Qian \\ Department of Electrical and Computer Engineering \\ TEES-AgriLife Center for Bioinformatics \& Genomic \\ Systems Engineering \\ Texas A\&M University \\ College Station, Texas \\ xqian@ece.tamu.edu
}

\begin{abstract}
Missing values frequently arise in modern biomedical studies due to various reasons, including missing tests or complex profiling technologies for different omics measurements. Missing values can complicate the application of clustering algorithms, whose goals are to group points based on some similarity criterion. A common practice for dealing with missing values in the context of clustering is to first impute the missing values, and then apply the clustering algorithm on the completed data. The performance of such methods, however, depends on the knowledge of missing value mechanism, which is rarely fully achievable in practice. We consider missing values in the context of optimal clustering, which finds an optimal clustering operator with reference to an underlying random labeled point process (RLPP). We present how the missing-value problem fits neatly into the overall framework of optimal clustering by marginalizing out the missing-value process from the feature distribution. In particular, we demonstrate the proposed framework for the multivariate Gaussian model with an arbitrary covariance structure. Comprehensive experimental studies on both synthetic and real-world RNA-seq data shows the superior performance of the proposed optimal clustering with missing values, compared to various clustering approaches, including $k$-means, fuzzy $c$-means and hierarchical clustering, with the off-the-shelf Gibbs sampling based imputation method. Optimal clustering offers a robust and flexible framework for dealing with the missing value problem, obviating the need for imputation-based pre-processing of the data. Its superior performance compared to various clustering methods in settings with different missing rates and small sample sizes,
\end{abstract}

\footnotetext{
*Equal contributor

${ }^{\dagger}$ Corresponding author

Permission to make digital or hard copies of part or all of this work for personal or classroom use is granted without fee provided that copies are not made or distributed for profit or commercial advantage and that copies bear this notice and the full citation on the first page. Copyrights for third-party components of this work must be honored. For all other uses, contact the owner/author(s).

ACM-BCB'18, August 29-September 1, 2018, Washington, DC, USA

(C) 2018 Copyright held by the owner/author(s).

ACM ISBN 978-1-4503-5794-4/18/08.

https://doi.org/10.1145/3233547.3233687
}

\author{
Siamak Zamani Dadaneh* \\ Department of Electrical and Computer Engineering \\ Texas A\&M University \\ College Station, Texas \\ siamak@tamu.edu
}

Edward R. Dougherty ${ }^{\dagger}$

Department of Electrical and Computer Engineering

TEES-AgriLife Center for Bioinformatics \& Genomic

Systems Engineering

Texas A\&M University

College Station, Texas

edward@ece.tamu.edu

demonstrates the optimal clusterer as a promising tool for dealing with missing data in biomedical applications.

\section{CCS CONCEPTS}

- Mathematics of computing $\rightarrow$ Cluster analysis; • Computing methodologies $\rightarrow$ Cluster analysis; $\bullet$ Applied computing $\rightarrow$ Bioinformatics; Computational genomics; • Theory of computation $\rightarrow$ Unsupervised learning and clustering;

\section{KEYWORDS}

Clustering; missing data; optimal design; pattern recognition

\section{ACM Reference Format:}

Shahin Boluki, Siamak Zamani Dadaneh, Xiaoning Qian, and Edward R. Dougherty. 2018. Optimal Clustering with Missing Values. In ACM-BCB'18: 9th ACM International Conference on Bioinformatics, Computational Biology and Health Informatics, August 29-September 1, 2018, Washington, DC, USA. ACM, New York, NY, USA, 2 pages. https://doi.org/10.1145/3233547.3233687

\section{RESULTS}

The performance of the proposed clustering with missing values framework is examined on a publicly available RNA-seq dataset of breast cancer. The data is available from The Cancer Genome Atlas (TCGA) [8], and is procured by the R package TCGS2STAT [9]. It consists of matched tumor and normal samples, and includes 97 points from each. The original data are in terms of the number of sequence reads mapped to each gene. RNA-seq data are integers, highly skewed and over-dispersed [3,4]. Thus, we apply a variance stabilizing transformation (VST) [6] implemented in DESeq2 package [7], and transform data to a $\log 2$ scale that have been normalized with respect to the library size. For all subsequent analysis, other than for calculating clustering errors, we assume that the labels of data are unknown. Feature selection is performed in a completely unsupervised manner, since in clustering no labels are known in practice. The top 10 genes in terms of variance to mean ratio of expression are picked as features to be used in clustering algorithms. In general, for setting prior hyperparameters, external sources of information like signaling pathways, where available, can be leveraged $[1,2]$. Here, we only use a subset of the discarded gene expressions, i.e. the next 90 top genes (in terms of variance to 
mean ratio of expression), for prior hyperparameters calibration for the optimal/suboptimal approaches. We follow the approach in [5] and use the method of moments for prior calibration, but unlike [5], a single set of hyperparameters are estimated and used for both clusters, since the labels of data are not available. It is well known that in small sample size settings, estimation of covariance matrices, scatter matrices and even mean vectors may be problematic. Therefore, similar to [5], we assume the following structure

$$
\begin{aligned}
& \Psi_{0}=\Psi_{1}=\left[\begin{array}{cccc}
\sigma^{2} & \rho \sigma^{2} & \ldots & \rho \sigma^{2} \\
\rho \sigma^{2} & \sigma^{2} & \cdots & \rho \sigma^{2} \\
\vdots & \vdots & \ddots & \vdots \\
\rho \sigma^{2} & \cdots & \cdots & \sigma^{2}
\end{array}\right]_{d \times d}, \\
& m_{0}=m_{1}=m[1, \cdots, 1]_{d}^{T}, \\
& v_{0}=v_{1}=v, \kappa_{0}=\kappa_{1}=\kappa,
\end{aligned}
$$

and estimate five scalars $\left(m, \sigma^{2}, \rho, \kappa, v\right)$ from the data.

In each repetition, stratified sampling is done, i.e. $n_{1}$ and $n_{2}$ points are sampled randomly from each group (normal and tumor). When $n_{1} \neq n_{2}$, in half of the repetitions $n_{1}$ and $n_{2}$ points are randomly selected from the normal and tumor samples, respectively, and vice-versa in the other half. Prior calibration is performed in each repetition, and $15 \%$ of the features are considered as missing values. The clustering error of each method in each iteration is calculated by comparing the predicted labels and true labels of the sampled points (with accounting for label switching issue), and the average results over 40 repetitions are provided in Figure 1. It can be seen that the proposed optimal clustering with missing values and its suboptimal versions (Pmax and Pseed) outperform the classical algorithms.

Our methodology is very natural. As with any signal processing problem, the basic problem is to find an optimal operator from a class of operators given the underlying random process and a cost function, which is often an error associated with operator performance. While it may not be possible to compute the optimal operator, one can at least employ suboptimal approximations to it while knowing the penalties associated with the approximations. In the present situation involving clustering, in the standard imputation-followed-by-clustering approach, it is typically the case that neither the imputation (filter) nor the clustering (decision) is optimal, so that even more advantage is obtained by optimal clustering over the missing-value-adjusted RLPP. Optimal clustering with missing values obviates the need for imputation-based preprocessing of the data, while at the same time possessing smaller clustering errors.

\section{REFERENCES}

[1] S. Boluki, M. Shahrokh Esfahani, X. Qian, and E. R. Dougherty. 2017. Constructing Pathway-based Priors Within a Gaussian Mixture Model for Bayesian Regression and Classification. IEEE/ACM Transactions on Computational Biology and Bioinformatics (2017), 1-1. https://doi.org/10.1109/TCBB.2017.2778715

[2] Shahin Boluki, Mohammad Shahrokh Esfahani, Xiaoning Qian, and Edward R. Dougherty. 2017. Incorporating biological prior knowledge for Bayesian learning via maximal knowledge-driven information priors. BMC Bioinformatics 18, 14 (28 Dec 2017), 552. https://doi.org/10.1186/s12859-017-1893-4

[3] Siamak Zamani Dadaneh, Xiaoning Qian, and Mingyuan Zhou. 2018. BNP-Seq: Bayesian nonparametric differential expression analysis of sequencing count data. 7. Amer. Statist. Assoc. 113, 521 (2018), 81-94.

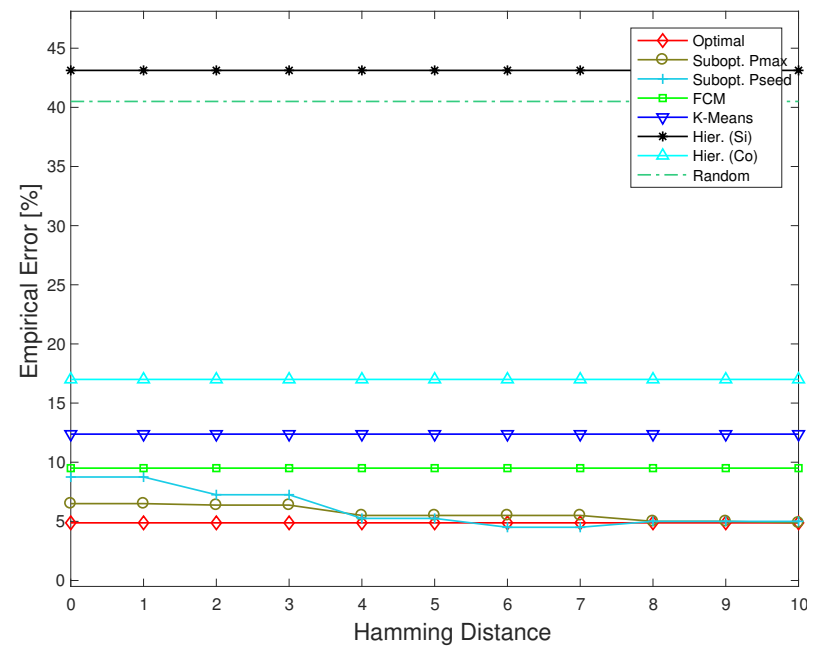

(a) $n_{1}=10, n_{2}=10$, miss. prob. $=0.15$

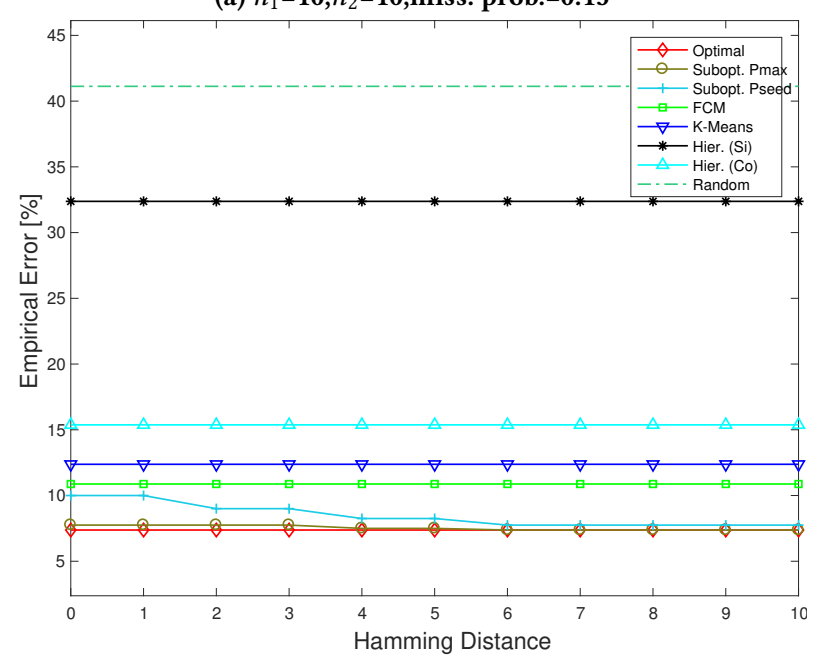

(b) $n_{1}=12, n_{2}=8$,miss. prob. $=0.15$

Figure 1: Empirical clustering errors on the TCGA dataset.

[4] Siamak Zamani Dadaneh, Mingyuan Zhou, and Xiaoning Qian. 2018. Bayesian negative binomial regression for differential expression with confounding factors. Bioinformatics 1 (2018), 8.

[5] Lori A Dalton and Edward R Dougherty. 2011. Application of the Bayesian MMSE estimator for classification error to gene expression microarray data. Bioinformatics 27, 13 (2011), 1822-1831.

[6] Blythe P Durbin, Johanna S Hardin, Douglas M Hawkins, and David M Rocke. 2002. A variance-stabilizing transformation for gene-expression microarray data. Bioinformatics 18, suppl_1 (2002), S105-S110.

[7] Michael I Love, Wolfgang Huber, and Simon Anders. 2014. Moderated estimation of fold change and dispersion for RNA-seq data with DESeq2. Genome Biology 15, 12 (2014), 550

[8] The Cancer Genome Atlas Research Network (TCGA). 2008. Comprehensive genomic characterization defines human glioblastoma genes and core pathways. Nature 455, 7216 (2008), 1061.

[9] Ying-Wooi Wan, Genevera I Allen, and Zhandong Liu. 2015. TCGA2STAT: simple TCGA data access for integrated statistical analysis in R. Bioinformatics 32, 6 (2015), 952-954. 